\title{
A FORMULA FOR DISTRIBUTION TRACE CHARACTERS ON NILPOTENT LIE GROUPS
}

\author{
L. CORWIN AND F. P. GREENLEAF
}

\begin{abstract}
The distribution trace character $\theta_{\pi}$ of an irreducible representation $\pi$ on a simply connected nilpotent Lie group $N$ is described as a superposition of unitary characters on certain subgroups, in terms of the canonical objects introduced by $R$. Penney $[8]$. For $l \in \mathfrak{n}^{*}$, define $\mathfrak{h}_{1}(l)=$ smallest ideal containing the radical $r(l)$, and $\mathfrak{h}_{h+1}(l)=\mathfrak{h}_{1}\left(l \mid \mathfrak{h}_{h}(l)\right)$. These subalgebras terminate in a subordinate subalgebra $\mathfrak{h}_{x}(l)$ after finitely many steps. If $H_{x}=\exp \left(\mathfrak{h}_{x}\right), \chi_{x}=\left(e^{2 \pi i l}\right) \circ \log \mid H_{x}$, and $\left(\chi_{x}, H_{x}\right) \cdot n=\left(\chi_{x} \cdot n, H_{x} \cdot n\right)$, where $H_{x} \cdot n=n^{-1} H_{x} n, \chi_{x} \cdot n\left(h^{\prime}\right)=$ $\chi_{\infty}\left(n h^{\prime} n^{-1}\right)$ on $H_{\infty} \cdot n$, then $\left\langle\theta_{\pi}, \phi\right\rangle=\int_{K_{x} \backslash N}\left\langle\left(\chi_{x}, H_{x}\right) \cdot n, \phi\right\rangle d \dot{n}$, where the pair $\left(\chi_{x}, H_{x}\right) \cdot n$ is regarded as the tempered distribution $\left\langle\left(\chi_{x}, H_{x}\right) \cdot n, \phi\right\rangle=$ $\int_{\|_{\infty}} \chi_{x}(h) \phi\left(n^{-1} h n\right) d h$, and where ${ }^{*}{ }_{x}=\left\{X \in n: l\left[h_{x}, X\right]=0\right\}$ gives the stabilizer of the pair $\left(\chi_{x}, H_{x}\right)$. The integral over $K_{x} \backslash N$ is absolutely convergent for any Schwartz function $\phi$ on $N$.
\end{abstract}

If $N$ is a simply connected nilpotent Lie group and $\pi \in \hat{N}$ an irreducible representation, then $\pi_{\phi}$ is trace class for any $\phi \in C_{c}^{\infty}(N)$ and the trace character $\left\langle\theta_{\pi}, \phi\right\rangle=\operatorname{Tr} \pi_{\phi}$ is a tempered distribution on $N$. Its Fourier transform has been described in terms of coadjoint orbits in $n^{*}$ by Kirillov [6]. Various attempts have been made to describe the $\theta_{\pi}$ directly as distributions on $N$, but this seems quite difficult. Dixmier [3] computed a number of examples, and some asymptotic properties were discussed in [1] for special $N$.

This note shows how $\theta_{\pi}$ can be represented as a superposition of ordinary characters on certain canonical subgroups on $N$ related to the canonical objects introduced by Penney in [8], and exploited in [2]. The formula we get is clearly suggested by [2], but a very direct calculation can be made for the $\theta_{\pi}$. This formula can be used to estimate the order of $\theta_{\pi}$ as a Schwartz distribution, and may prove to have uses in the harmonic analysis of smooth functions on $N$.

Let $\delta(N)$ be the Schwartz functions on $N$ (i.e. $f \circ \exp$ is Schwartz on the Lie algebra $n$ ). If $\pi \in \hat{N}$, let $l$ be an element in the coadjoint orbit $\theta=\theta_{\pi} \subseteq n^{*}, m$ a maximal subordinate subalgebra for $l$, and $\chi=\left(e^{2 \pi i l}\right) \circ \log \mid M$ the resulting character on $M=\exp (\mathrm{m})$. Let $\left\{X_{1}, \ldots, X_{m}, \ldots, X_{n}\right\}$ be a Jordan-Hölder basis for $n$ that passes through $\mathrm{m} ; \mathfrak{n}_{j}=\mathbf{R}-\operatorname{span}\left\{X_{1}, \ldots, X_{j}\right\}$ a subalgebra with $\mathfrak{n}_{j} \triangleleft \mathfrak{n}_{j+1}$ for all $j$, and $\mathfrak{n}_{m}=\mathfrak{m}$. The map $\gamma: \mathbf{R}^{k} \rightarrow N$,

$$
\gamma(t)=\exp \left(t_{1} X_{m+1}\right) \cdots \exp \left(t_{k} X_{m+k}\right), \quad k=n-m,
$$

Received by the editors August 20, 1982 and, in revised form, March 21, 1983.

1980 Mathematics Subject Classification. Primary 22E27. 
cross sections the $M \backslash N$ cosets, carries Lebesgue measure $d t$ to right invariant measure $d \dot{n}$ on $M \backslash N$, and allows us to realize the Hilbert space of the induced representation $\pi=\operatorname{Ind}(M \uparrow N, \chi)$ isometrically as $L^{2}\left(\mathbf{R}^{k}\right)$, as follows: the function $\tilde{f}$ on $G$ corresponds to $f \in L^{2}\left(\mathbf{R}^{k}\right)$ via restriction to the cross-section $\gamma\left(\mathbf{R}^{k}\right)$ and pullback to $\mathbf{R}^{k}$,

$$
\tilde{f}(n)=\tilde{f}(m \gamma(t))=\chi(m) f(t) .
$$

For $\phi \in S(N)$ we may explicitly compute the kernel $K_{\phi}(s, t)$ of the operator $\pi_{\phi}$ :

$$
\begin{aligned}
\pi_{\phi} f(t) & =\pi_{\phi} \tilde{f}(\gamma(t))=\int_{N} \phi(n) \pi_{n} \tilde{f}(\gamma(t)) d n=\int_{N} \phi\left(\gamma(t)^{-1} n\right) \tilde{f}(n) d n \\
& =\int_{\mathbf{R}^{k}} \int_{M} \phi\left(\gamma(t)^{-1} m \gamma(s)\right) \tilde{f}(m \gamma(s)) d m d s \\
& =\int_{\mathbf{R}^{k}}\left[\int_{M} \chi(m) \phi\left(\gamma(t)^{-1} m \gamma(s)\right) d m\right] f(s) d s \\
& =\int_{\mathbf{R}^{k}} K_{\phi}(t, s) f(s) d s .
\end{aligned}
$$

Since $\pi_{\phi}$ is trace class $[9$, p. $108 \mathrm{ff}$.$] and K_{\phi}$ is continuous, the trace is just the integral of the diagonal values

$$
\left\langle\theta_{\pi}, \phi\right\rangle=\operatorname{Tr} \pi_{\phi}=\int_{\mathbf{R}^{k}} K_{\phi}(t, t) d t
$$

and the integral is absolutely convergent (see [4, III.10] for example).

Let $(\chi, M) \cdot n=(\chi \cdot n, M \cdot n)$, where $M \cdot n=n^{-1} M n$ and $\chi \cdot n\left(m^{\prime}\right)=\chi\left(n m^{\prime} n^{-1}\right)$ for $m^{\prime} \in M \cdot n$, to obtain a right action of $N$ on pairs $(\chi, M)$ where $\chi$ is a character on $M$. Fixing a Haar measure $d m$ on $M$, we may regard each pair in an $N$ orbit as a tempered distribution

$$
\langle(\chi, M) \cdot n, \phi\rangle=\int_{M} \chi(m) \phi\left(n^{-1} m n\right) d m, \quad \text { all } \phi \in \delta(N) .
$$

From (1) and the fact that $(\chi, M)=(\chi, M) \cdot n \Leftrightarrow n \in M$, we see that $\theta_{\pi}$ is a superposition of all pairs in the orbit $(\chi, M) \cdot N$ :

$$
\begin{aligned}
\left\langle\theta_{\pi}, \phi\right\rangle & =\int_{\mathbf{R}^{k}} K_{\phi}(t, t) d t=\int_{\mathbf{R}^{k}}\left[\int_{M} \chi(m) \phi\left(\gamma(t)^{-1} m \gamma(t)\right) d m\right] d t \\
& =\int_{M \backslash N}\langle(\chi, M) \cdot n, \phi\rangle d \dot{x},
\end{aligned}
$$

the formula being absolutely convergent for all $\phi \in S(N)$. Formula (2) is not canonical since it involves a choice of $l$ and a polarization $\mathrm{m}$. It must involve a great deal of hidden cancellation, as the canonical version below indicates.

For $l \in \mathrm{n}^{*}$ we define, following Penney [8], the canonical objects: $\mathfrak{h}_{1}(l)=$ smallest ideal containing the radical $\mathfrak{r}(l)$, and $\mathfrak{h}_{k+1}(l)=\mathfrak{h}_{1}\left(l \mid \mathfrak{h}_{k}(l)\right)$. These subalgebras stabilize eventually, $\mathfrak{h}_{1} \supseteq \mathfrak{h}_{2} \supseteq \cdots \supseteq \mathfrak{h}_{k}=\mathfrak{h}_{k+1}=\cdots \mathfrak{h}_{\infty}$; all contain the radical 
$r(l)$ and are transformed covariantly:

$$
\mathfrak{h}_{k}(l \cdot n)=\mathfrak{h}_{k}(l) \cdot n \quad \text { where } l \cdot n=\operatorname{Ad}^{*}\left(n^{-1}\right) l \text { and } X \cdot n=\operatorname{Ad}\left(n^{-1}\right) X,
$$

and $\mathfrak{h}_{\infty}$ is subordinate to $l$. Let $\mathfrak{f}_{\infty}(l)=\left\{X \in \mathfrak{n}: l\left[\mathfrak{h}_{\infty}, X\right]=0\right\}$; this turns out to be a subalgebra, and if we set $l_{\infty}=l \mid \mathfrak{f}_{\infty}$, then $\mathfrak{r}\left(l_{\infty}\right)=\mathfrak{h}_{\infty}(l) \triangleleft \mathfrak{f} \mathfrak{c}_{\infty}(l)$ so that $l_{\infty} \in \mathfrak{f}_{\infty}^{*}$ has flat orbit (see [7]). Let $\chi_{\infty}=\left(e^{2 \pi i l}\right) \circ \log \mid H_{\infty}$; by fixing a Haar measure $d h$ on $H_{\infty}$ we may regard $\left(\chi_{\infty}, H_{\infty}\right)$ and all its conjugates $\left(\chi_{\infty}, H_{\infty}\right) \cdot n$ as tempered distributions. The significance of the following formula is that $\mathfrak{h}_{\infty}$ can be much smaller than any polarization $\mathrm{m}$ for $l$.

Theorem 1. Let $l \in \mathfrak{n}^{*}$ and define $H_{\infty}, K_{\infty}, \chi_{\infty}$ as above. Fixing dh on $H_{\infty}$ and $d \dot{n}$ on $K_{\infty} \backslash N$, and regarding each $\left(\chi_{\infty}, H_{\infty}\right) \cdot n$ as a tempered distribution

$$
\left\langle\left(\chi_{\infty}, H_{\infty}\right) \cdot n, \phi\right\rangle=\int_{H_{\infty}} \chi(h) \phi\left(n^{-1} h n\right) d h, \quad \phi \in S(N),
$$

we get

$$
\left\langle\theta_{\pi}, \phi\right\rangle=\int_{K_{\infty} \backslash N}\left\langle\left(\chi_{\infty}, H_{\infty}\right) \cdot n, \phi\right\rangle d \dot{n}, \quad \text { all } \phi \in \mathcal{S}(N),
$$

where din is suitably normalized. The integral is absolutely convergent.

Proof. Recall that $K_{\infty}=\left\{x \in N:\left(\chi_{\infty}, H_{\infty}\right) \cdot n=\left(\chi_{\infty}, H_{\infty}\right)\right\}$ (see [2]). Let $M$ be any maximal subordinate subgroup for $l_{\infty} \in \mathfrak{H}_{\infty}^{*}$; it must also be maximal subordinate for $l \in \mathrm{n}^{*}$, by dimension counting. Let $\chi=\left(e^{2 \pi i l}\right) \circ \log \mid M$ and $\pi=$ $\operatorname{Ind}(M \uparrow N, \chi)$. We start with a remark on the special case: $\pi$ square integrable modulo $Z(N)$. This is precisely the case in which $H_{\infty}(l)=R(l)=Z(N)$. As is well known [7], we have flat orbit $\theta(l)=l+z^{\perp}$. From a simple version of Kirillov's orbital integral formula and Poisson summation, we get

$$
\begin{aligned}
\theta_{\pi} & =\text { Euclidean Fourier transform of Lebesgue measure on } l+z^{\perp} \\
& =\text { scalar multiple of } \chi_{\infty} \text { weighting Haar measure on } Z(N),
\end{aligned}
$$

or $\left\langle\theta_{\pi}, \varphi\right\rangle=\left\langle\left(\chi_{\infty}, H_{\infty}\right), \varphi\right\rangle$ where $d h$ on $H_{\infty}$ is suitably chosen. Since $\mathfrak{f}_{\infty}=\mathfrak{n}$ here, we have proved (3) in this special case.

We now use the noncanonical formula (2) to complete the proof of (3).

Take an arbitrary $l \in \mathrm{n}^{*}$, consider a system $H_{\infty} \subseteq M \subseteq K_{\infty}$, and let

$$
\pi_{\infty}=\operatorname{Ind}\left(M \uparrow K_{\infty}, \chi\right) \in \hat{K_{\infty}} .
$$

Applying (2) to this situation, we get

$$
\left\langle\theta_{\pi_{\infty}}, \phi\right\rangle=\int_{M \backslash K_{\infty}}\langle(\chi, M) \cdot k, \phi\rangle d \dot{k}, \quad \text { all } \phi \in \mathcal{S}\left(K_{\infty}\right)
$$

(or $\phi \in S(N)$, for that matter). We claim that

$$
\left\langle\theta_{\pi_{\infty}}, \phi\right\rangle=\left\langle\left(\chi_{\infty}, H_{\infty}\right), \phi\right\rangle, \quad \text { all } \phi,
$$


so the superposed conjugates of $(\chi, M)$ by elements in $K_{\infty}$ collapse to the single character $\left(\chi_{\infty}, H_{\infty}\right)$ through cancellations. Once (5) is proved, we finish proving (3) as follows: by Fubini,

$$
\begin{aligned}
\left\langle\theta_{\pi}, \phi\right\rangle & =\int_{M \backslash N}\langle(\chi, M) \cdot n, \phi\rangle d \dot{n} \\
& =\int_{K_{\infty} \backslash N}\left[\int_{M \backslash K_{\infty}}\langle(\chi, M) \cdot k, \phi \circ \operatorname{Ad}(x)\rangle d \dot{k}\right] d \dot{x} \\
& =\int_{K_{\infty} \backslash N}\left\langle\left(\chi_{\infty}, H_{\infty}\right), \phi \circ \operatorname{Ad}(x)\right\rangle d \dot{x}=\int_{K_{\infty} \backslash N}\left\langle\left(\chi_{\infty}, H_{\infty}\right) \cdot x, \phi\right\rangle d \dot{x}
\end{aligned}
$$

with all integrals absolutely convergent.

To prove (5) it suffices to prove the following general statement about functionals $l \in \mathrm{n}^{*}$ with flat orbit (i.e. $\mathrm{r}(l) \triangleleft \mathrm{n}$, so that $H_{\infty}=R(l), K_{\infty}=N$ and $\pi_{\infty}=\pi=$ $\operatorname{Ind}(M \uparrow N, \chi))$.

(6) If $l \in \mathfrak{n}^{*}$ has $\mathrm{r}(l) \triangleleft \mathfrak{n}, \mathfrak{m}$ is any maximal subordinate subalgebra, $\chi=$ $\left(e^{2 \pi i l}\right) \circ \log \mid M$, and $\pi=\operatorname{Ind}(M \uparrow N, \chi)$, then $\theta_{\pi}=\left(\chi_{\infty}, H_{\infty}\right)$.

Here the orbit is flat, and $\theta_{\pi}=l+\mathcal{K}_{\infty}^{\perp}$; the $N$-invariant measure on $\theta_{\pi}$ is just Euclidean measure on $\mathcal{H}_{\infty}^{\perp}$ lifted to this coset. By Poisson summation and Kirillov's description of $\theta$, we get (6). This completes the proof of Theorem 1. Q.E.D.

The canonical formula (3) leads to the following conclusion about flat parts in coadjoint orbits. If $m$ is maximal subordinate for $l \in n^{*}$, it is well known that $\theta$ is a disjoint union of the flat fibers $l \cdot x+m^{\perp} \cdot x=l \cdot M x, \dot{x} \in M \backslash N$. In general $\mathfrak{h}_{\infty} \subseteq \mathfrak{m}$, so $\mathfrak{h}_{\infty}^{\perp}$ may be larger than $\mathfrak{m}^{\perp}$. From (3) we see that

$$
\vartheta=\cup\left\{\left(l+\mathfrak{h}_{\infty}^{\perp}\right) \cdot x: x \in K_{\infty} \backslash N\right\} .
$$

This is a disjoint union: if $x, y \in N$ give nondisjoint fibers, then $\left(l+\mathfrak{h}_{\infty}^{\perp}\right) \cdot x y^{-1}$ meets $l+\mathfrak{h}_{\infty}^{\perp}$. But by dimension counting we can show that $l+\mathfrak{h}_{\infty}^{\perp}=l \cdot K_{\infty}$, so there exist $k_{1}, k_{2} \in K_{\infty}$ such that $l \cdot k_{1}\left(x y^{-1}\right) k_{2}=l$. Thus $k_{1}\left(x y^{-1}\right) k_{2} \in R(l) \subseteq H_{\infty}$ $\subseteq K_{\infty}$, and $x K_{\infty}=y K_{\infty}$ as required. Formula (3) also shows that $\operatorname{supp}\left(\theta_{\pi}\right) \subseteq$ the smallest connected (normal) subgroup containing $\cup_{n \in N} n^{-1} H_{\infty} n$. Since $\mathfrak{h}_{1}=$ ideal generated by $r(l) \supseteq \mathfrak{h}_{\infty} \supseteq \mathrm{r}(l)$, this amounts to saying that $\operatorname{supp}\left(\theta_{\pi}\right) \subseteq H_{1}$. By other means, Pukanszky [10] has recently shown that a normal Lie subgroup $H$ contains $\operatorname{supp}\left(\theta_{\pi}\right) \Leftrightarrow H \supseteq H_{1}$, so further reduction of the support through cancellation cannot occur.

\section{REFERENCES}

1. L. Corwin and F. P. Greenleaf, Singular Fourier integral operators and representations of nilpotent Lie groups, Comm. Pure Appl. Math. 31 (1978), 681-7ns

2. L. Corwin, F. P. Greenleaf and R. Penney, A canonical formula for the distribution kernels of primary projections in $L^{2}$ of a nilmanifold, Comm. Pure Appl. Math. 30 (1977), 355-372.

3. J. Dixmier, Sur les représentations unitaires des groupes de Lie nilpotents. IV, Canad. J. Math. 11 (1959), 321-344.

4. I. C. Gohberg and M. G. Krein, Introduction to the theory of linear nonselfadjoint operators, Transl. Math. Mono., vol. 18, Amer. Math. Soc., Providence, R. I., 1969. 
5. R. Howe, On a connection between nilpotent groups and oscillatory integrals associated to singularities, Pacific J. Math. 73 (1977), 329-364.

6. A. A. Kirillov, Unitary representations of nilpotent Lie groups, Uspehi Mat. Nauk 17 (1962), 57-110.

7. C. C. Moore and J. Wolf, Square integrable representations of nilpotent groups. Trans. Amer. Math. Soc. 185 (1973), 445-462.

8. R. Penney, Canonical objects in the Kirillow theory of nilpotent Lie groups, Proc. Amer. Math. Soc. 66 (1977), 175-178.

9. L. Pukanszky, Leçons sur les représentations des groupes, Dunod, Paris, 1967.

10. __. On Kirillow's character formula, J. Reine Angew. Math. 311/312 (1979), 408-440.

Department of Mathematics, Rutgers University, New Brunswick, NeW Jersey 08903

Courant Institute of Mathematical Sciences, New York University, New York, New York 10012 